INPLASY

PROTOCOL

To cite: Ma et al. Effects of exercise in preventing cardiotoxicity in breast cancer patients receiving chemotherapy: a systematic review. Inplasy protocol

2021110085. doi:

10.37766/inplasy2021.11.0085

Received: 23 November 2021

Published: 23 November 2021

Corresponding author:

Fang Cheng

chfnancy@163.com

Author Affiliation:

School of Nursing, Nanjing

Medical University

Support: National Natural

Science Found.

Review Stage at time of this submission: Formal screening of search results.

Conflicts of interest:

None declared.

\section{Effects of exercise in preventing cardiotoxicity in breast cancer patients receiving chemotherapy: a systematic review}

\author{
Ma, Z1; Yao, S; Cheng, F3; Shi, Y4; Ping, Z5; Lu, N6.
}

Review question / Objective: P: Breast cancer chemotherapy patients; I: Various forms of exercise; C: Routine Care; O: Cardiotoxicity and related test indicators; S: Randomized controlled trials, single-group before-and-after trials, longitudinal studies.

Condition being studied: Cardiotoxicity refers to the direct effects on cardiac function and structure following drug therapy (e.g. anthracyclines and trastuzumab) and is a recognized side effect of antineoplastic therapy. Exercise therapy is considered a viable non-pharmacological approach to manage several cardiovascular risk factors and a safe and effective supportive care for cancer survivors. In addition, exercise is considered a promising strategy for the prevention of cardiotoxicity associated with anticancer therapies. National and international studies have shown that exercise therapy has a mitigating effect on cardiotoxicity in breast cancer chemotherapy patients, including studies on the cardioprotective effects of exercise, but a systematic evaluation of exercise and its modalities on the prevention of cardiotoxicity is lacking. Therefore, the purpose of this systematic evaluation was to analyze the evidence regarding the outcomes associated with exercise therapy on cardiotoxicity.

INPLASY registration number: This protocol was registered with the International Platform of Registered Systematic Review and Meta-Analysis Protocols (INPLASY) on 23 November 2021 and was last updated on 23 November 2021 (registration number INPLASY2021110085).

\section{INTRODUCTION}

Review question / Objective: P: Breast cancer chemotherapy patients; I: Various forms of exercise; C: Routine Care; O:
Cardiotoxicity and related test indicators; S: Randomized controlled trials, singlegroup before-and-after trials, longitudinal studies. 
Condition being studied: Cardiotoxicity refers to the direct effects on cardiac function and structure following drug therapy (e.g. anthracyclines and trastuzumab) and is a recognized side effect of antineoplastic therapy. Exercise therapy is considered a viable nonpharmacological approach to manage several cardiovascular risk factors and a safe and effective supportive care for cancer survivors. In addition, exercise is considered a promising strategy for the prevention of cardiotoxicity associated with anticancer therapies. National and international studies have shown that exercise therapy has a mitigating effect on cardiotoxicity in breast cancer chemotherapy patients, including studies on the cardioprotective effects of exercise, but a systematic evaluation of exercise and its modalities on the prevention of cardiotoxicity is lacking. Therefore, the purpose of this systematic evaluation was to analyze the evidence regarding the outcomes associated with exercise therapy on cardiotoxicity.

\section{METHODS}

Participant or population: Breast cancer chemotherapy patients

Intervention: Various forms of exercise.

Comparator: Routine Care.

Study designs to be included: Randomized controlled trials, single-group before-andafter trials, longitudinal studies.

\section{Eligibility criteria: Inclusion Criteria:}

1)Participants: Studies that involving adult women (> 18 years old) with $\mathrm{BC}$, who were undergoing chemotherapy and performed concurrently a physical exercise intervention, were included. Studies which involved other cancer types beyond BC will be excluded.2)Interventions: Studies that involved aerobic training, resistance training and combined movement. 3)Comparators: non-exercise group (i.e. waiting list, control or placebo) 4)Outcome were eligible if a outcomeAssociated with cardiotoxicity, with no limitations in the measurement tools used. 5)Study type: Randomized controlled trials, single-group before-and-after trials, longitudinal studies Exclusion Criteria: 1)the studies were conference abstracts, study protocols, pilot studies, and duplicate reports 2)the studies did not contain complete data 3)the studies did not have relevant dataThere were no restrictions regarding the date of publication.

Information sources: We will use 9 database to search all relevant literature resources.Including five English databases PubMed, Embase, Web of Science, Cochrane, CINAHL, and four Chinese d a t abases China Biomedical Literature(CBM), the Wanfang Chinese digital periodical and conference database(WangFang Data), China National Knowledge Infrastructure database(CNKI), and the VIP Chinese Science and Technique Journals Database(VIP). The retrieval time starts from them until November 2021. Key words include "Exercise", "Breast cancer", "Cardiotoxicity", "Systematic review", "Meta-analysis". We will also search for ongoing or unpublished trials from the National Institutes of Health (NIH) clinical registration clinical trials and the China clinical trial registration platform.

Main outcome(s): LVEF(Left ventricular ejection fraction); VO2max(maximal oxygen uptake).

Additional outcome(s): BNP(brain natriuretic peptide); GLS(global longitudinal strain), E/A(), CK-MB, Troponin.

Quality assessment / Risk of bias analysis: The two authors will independently assess and score the methodological quality of the included trials using the Cochrane Collaboration's Risk of Bias Assessment Tool for random sequence generation, allocation concealment, blinding of participants and personnel, blinding of outcome assessments, incomplete outcome data, selective reporting, and other potential sources of bias, with the 
level of risk of bias determined for each domain. (1) high risk, (2) unclear risk, (3) low risk. Disagreements, if they arise, should be resolved by discussion and, if necessary, by consultation with a third author.

Strategy of data synthesis: Data will be managed using RevMan version 5.3. Statistical heterogeneity will be tested by the 2 test and quantified by the 12 statistic. Heterogeneity will be assumed if we verified a $p$ value of $<0.1$ for 2 and 12 of $\geq$ $50 \%$. We will perform sensitivity analyses stratified according to the risk of bias. If heterogeneity regarding design and comparators is found in most studies, a meta-analysis of random effects models will be performed. For continuous outcomes, we plan to estimate standardized mean differences (SMD) with a $95 \%$ confidence interval (Cl 95\%).

Subgroup analysis: If necessary, we will conduct a subgroup analysis based on the type of intervention (aerobic or resistance, concomitant or isolated), exercise intensity (light, moderate, vigorous), treatment scheme (including anthracyclines or trastuzumab, concomitant or isolated).

Sensitivity analysis: Considering that the diversity of included studies will lead to a certin degree of heterogeneity and inconsistency, we will conduct a sensitivity analysis. The studies were exclued one by one, and then the meta-analysis was performed in the remaining studies. If the heterogeneity does not change after excluding each literature, we think our conclusion is stable; otherwise, if the heterogeneity changes, the excluded literature may be the source of heterogeneity.

Language: Chinese and English.

Country(ies) involved: China.

Keywords: exercise; cardiotoxicity; breast cancer; systematic review; meta-analysis.

Contributions of each author:

Author 1 - Zhuyue Ma.
Author 2 - Shanshan Yao.

Author 3 - Fang Cheng.

Author 4 - Yanyan Shi.

Author 5 - Ping Zhu.

Author 6 - Ningning Lu. 